\title{
Stability results for logarithmic Sobolev and Gagliardo-Nirenberg inequalities
}

\author{
Jean Dolbeault ${ }^{1}$ and Giuseppe Toscani $^{2}$ \\ ${ }^{1}$ Ceremade, UMR CNRS nr. 7534, Université Paris-Dauphine, Place de Lattre de Tassigny, 75775 \\ Paris Cedex 16, France, E-mail: dolbeaul@ceremade.dauphine.fr, \\ 2 Department of Mathematics, University of Pavia, via Ferrata 1, 27100 Pavia, Italy, \\ E-mail: giuseppe.toscani@unipv.it.
}

\begin{abstract}
This paper is devoted to improvements of functional inequalities based on scalings and written in terms of relative entropies. When scales are taken into account and second moments fixed accordingly, deficit functionals provide explicit stability measurements, i.e., bound with explicit constants distances to the manifold of optimal functions. Various results are obtained for the Gaussian logarithmic Sobolev inequality and its Euclidean counterpart, for the Gaussian generalized Poincaré inequalities and for the Gagliardo-Nirenberg inequalities. As a consequence, faster convergence rates in diffusion equations (fast diffusion, Ornstein-Uhlenbeck and porous medium equations) are obtained.
\end{abstract}

Keywords: Sobolev inequality; logarithmic Sobolev inequality; Gaussian isoperimetric inequality; generalized Poincaré inequalities; Gagliardo-Nirenberg inequalities; interpolation; entropy - entropy production inequalities; extremal functions; optimal constants; relative entropy; generalized Fisher information; entropy power; stability; improved functional inequalities; fast diffusion equation; Ornstein-Uhlenbeck equation; porous medium equation; rates of convergence

Mathematics Subject Classification (2010): 26D10; 46E35; 58E35

\section{Introduction}

Several papers have recently been devoted to improvements of the logarithmic Sobolev inequality. Ledoux et al. [2014] use the Stein discrepancy. Closer to our approach is Bobkov et al. [2014], Fathi et al. [2014], who exploit the difference between the inequality of [Stam, 1959, Inequality (2.3)] and the logarithmic Sobolev inequality to get a correction term in terms of the Fisher information functional. What we do here first is to emphasize the role of scalings and prefer to rely on Weissler [1978] for a scale invariant form of the logarithmic Sobolev inequality on the Euclidean space. We also make the choice to get a remainder term that involves the entropy functional and is very appropriate for stability issues. This allows us to deduce striking results in terms of rates of convergence for the Ornstein-Uhlenbeck equation. Writing the improvement in terms of the entropy has several advantages: contraints on the second moment are made clear, improvements can be extended to all generalized Poincaré inequalities for Gaussian measures, which interpolate between the Poincaré inequality and the logarithmic Sobolev inequality, and stability results with fully explicit constants can be stated: see for instance Corollary 3, with an explicit bound of the distance to the manifold of all Gaussian functions given in terms of the so-called deficit functional. This is, for the logarithmic Sobolev inequality, the exact analogue of the result of Bianchi and Egnell [1991] for Sobolev's inequality.

However, putting the emphasis on scalings has other advantages, as the method easily extends to a nonlinear setting. We are henceforth in a position to get improved entropy - entropy production inequalities associated with fast diffusion flows based on the scale invariant forms of the associated Gagliardo-Nirenberg inequalities, which cover a well-known family of inequalities that contain the logarithmic Sobolev inequality, and Sobolev's inequality as an endpoint. This is not a complete surprise because such improvements were known from Dolbeault and Toscani [2013] using detailed properties of the fast diffusion equation. By writing the entropy - entropy production inequality in terms of the relative entropy functional and a generalized Fisher information, we deduce from the scaling properties of Gagliardo-Nirenberg inequalities a correction term involving the square of the relative entropy, and this is much simpler than using the properties of the nonlinear flow. The method

August 27, 2021

(C) 2014 by the authors. This paper may be reproduced, in its entirety, for non-commercial purposes. 
also works in the porous medium case, which is new, provides clear evidences on the role of the second moment, and finally explains the fast rates of convergence in relative entropy that can be observed in the initial regime, away from Barenblatt equilibrium or self-similar states.

The reader interested in further considerations on improvements of the logarithmic Sobolev inequality is invited to refer to Ledoux et al. [2014] and Bobkov et al. [2014], Fathi et al. [2014] for probabilistic point of view and a measure of the defect in terms of Wasserstein's distance, and to Ledoux [2001] for earlier results. Much more can also be found in Bakry et al. [2014]. Not all Gagliardo-Nirenberg-Sobolev inequalities are covered by our remarks and we shall refer to Carlen et al. [2014] and references therein for the spectral point of view and its applications to the Schrödinger operator. The logarithmic Sobolev inequality in scale invariant form is equivalent to the Gaussian isoperimetric inequality: a study of the corresponding deficit can be found in Mossel and Neeman [2014]. In the perspective of information theory, we refer to Toscani [2013, 2014a] for a recent account on a concavity property of entropy powers that involves the isoperimetric inequality. It is not possible to quote all earlier related contributions but at least let us point two of them: the correction to the logarithmic Sobolev inequality by an entropy term involving the Wiener transform in [Carlen, 1991, Theorem 6], and the HWI inequality by Otto and Villani [2000].

Gagliardo-Nirenberg inequalities (see Gagliardo [1958], Nirenberg [1959]) have been related with fast diffusion or porous media equations in the framework of the so-called entropy methods by Del Pino and Dolbeault [2002]. Also see the papers by Carrillo and Toscani [2000], Otto [2001], Carrillo and Vázquez [2003] for closely related issues. The message is simple: optimal rates of convergence measured in relative entropy are equivalent to best constant in the inequalities written in entropy - entropy production form. Later improvements have been obtained on asymptotic rates of convergence by Blanchet et al. [2009], Bonforte et al. [2010], Dolbeault and Toscani [2011], Denzler et al. [2015]. A key observation of Dolbeault and Toscani [2011] is the fact that optimizing a relative entropy with respect to scales determines the second moment. This observation was then exploited by Dolbeault and Toscani [2013] to get a first explicit improvement in the framework of Gagliardo-Nirenberg inequalities. Notice that many papers on improved interpolation inequalities use the estimate of Bianchi and Egnell [1991] with the major drawback that the value of the constant is not known. As a consequence the improved inequality, faster convergence rates for the solution to the fast diffusion equation were obtained and a new phenomenon, a delay, was shown by Dolbeault and Toscani [2015a]. Inspired by Villani [2000], Savaré and Toscani [2014] studied the $p$-th Rényi entropy and observed that the corresponding isoperimetric inequality is a Gagliardo-Nirenberg inequality in scale invariant form. Various consequences for the solutions to the evolution equations have been drawn in Carrillo and Toscani [2014] and Dolbeault and Toscani [2015b], which are strongly related with the present paper but can all be summarized in a simple sentence: scales are important and a better adjustment than the one given by the asymptotic regime gives sharper estimates. The counterpart in the present paper is that taking into account the scale invariant form of the inequality automatically improves on the inequality obtained by a simple entropy - entropy production method. Let us give some explanations.

At a formal level, the strategy of our paper goes as follows. Let us consider a generalized entropy functional $\mathcal{E}$, which is assumed to be nonnegative, and a generalized Fisher information functional $\mathcal{I}$. We further assume that they are related by a functional inequality of the form

$$
\mathcal{I}-\lambda \mathcal{E} \geq 0
$$

We denote by $\lambda$ the optimal proportionality constant. If the inequality is not in scale invariant form, we will prove in various cases that there exists a convex function $\varphi$, leaving from $\varphi(0)=0$ with $\varphi^{\prime}(0)=\lambda$ such that $\mathcal{I} \geq \varphi(\mathcal{E})$. Hence we have found an improved functional inequality in the sense that

$$
\mathcal{I}-\lambda \mathcal{E} \geq \varphi(\mathcal{E})-\lambda \mathcal{E}=\psi(\mathcal{E})
$$

where $\psi(\mathcal{E})$ is nonnegative and can be used to measure the distance to the optimal functions. This is a stability result. The left hand side, which is called the deficit functional in the literature, is now controlled from below by a nonlinear function of the entropy functional. A precise distance can be obtained by the Pinsker-CsiszárKullback inequality, which is no more than a Taylor expansion at order two, and some generalizations.The key observation is that the optimization under scaling (in the Euclidean space) amounts to adjust the second moment (in the Euclidean space but also in spaces with finite measure, like the Gaussian measure, after some changes of variables).

At this point it is worth to emphasize the difference in our approach compared to the one of Bobkov et al. [2014] for the logarithmic Sobolev inequality. What the authors do is that they write the improved inequality as $\varphi^{-1}(\mathcal{I}) \geq \mathcal{E}$ and deduce that

$$
\mathcal{I}-\lambda \mathcal{E} \geq \mathcal{I}-\lambda \varphi^{-1}(\mathcal{I})
$$


where the right hand side is again nonnegative because $\varphi^{-1}$ is concave and $\lambda\left(\varphi^{-1}\right)^{\prime}(0)=1$. This is of course a stronger form of the inequality, as it controls the distance to the manifold of optimal functions in a stronger norm, for instance. However, it is to a large extend useless for the applications that are presented in this paper, as the estimate in terms of the entropy is what matters, for instance, for application in evolution equations.

We shall apply our strategy to the logarithmic Sobolev inequality in Section 2, to the generalized Poincaré inequalities for Gaussian measures in Section 3 and to some Gagliardo-Nirenberg inequalities in Section 4. Each of these inequalities can be established by the entropy - entropy production method. By considering the Ornstein-Uhlenbeck equation in the first two cases, and the fast diffusion / porous medium equation in the third case, it turns out that $\frac{d \mathcal{E}}{d t}=-\mathcal{I}$ and

$$
-\frac{d}{d t}(\mathcal{I}-\lambda \mathcal{E})=\mathcal{R} \geq 0
$$

Hence, if $\lim _{t \rightarrow \infty}(\mathcal{I}-\lambda \mathcal{E})=0$, this shows with no additional assumption that $\int_{0}^{\infty} \mathcal{R}[v(t, \cdot)] d t$ is a measure of the distance to the optimal functions. Improved functional inequalities follow by ODE techniques if one is able to relate $\mathcal{R}$ with $\mathcal{E}$ and $\mathcal{I}$. This is the method which has been implemented for instance in Arnold and Dolbeault [2005], Dolbeault et al. [2008], Dolbeault and Toscani [2013] and it is well adapted when the diffusion equation can be seen as the gradient flow of $\mathcal{E}$ with respect to a distance. Typical distances are the Wasserstein distance for the logarithmic Sobolev inequality or the Gagliardo-Nirenberg inequalities, and ad hoc distances in case of the generalized Poincaré inequalities. See Jordan et al. [1998], Otto [2001], Dolbeault et al. [2009, $2012]$ for more details on gradient flow issues. Improvements can also be obtained when $\frac{d \mathcal{E}}{d t}$ differs from $-\mathcal{I}$ : we refer to Demange [2008], Dolbeault et al. [2014] for interpolation inequalities on compact manifolds, or to Dolbeault and Jankowiak [2014] for improvements of Sobolev's inequality based on the Hardy-LittlewoodSobolev functional. This makes the link with the famous improvement obtained by Bianchi and Egnell [1991], and also Cianchi et al. [2009], but so far no entropy - entropy production method has been able to provide an improvement in such a critical case. For completeness, let us mention that other methods can be used to obtain improved inequalities, which are based on variational methods like in Bianchi and Egnell [1991], on symmetrization techniques like in Cianchi et al. [2009] or on spectral methods connected with heat flows like in Arnold et al. [2007]. Here we shall simply rely on convexity estimates and the interplay of entropy - entropy production inequalities with their scale invariant counterparts.

A very interesting feature of improved functional inequalities in the framework of the entropy - entropy production method is that the entropy decays faster than expected by considering only the asymptotic regime. In that sense, the improved inequality capture an initial rate of convergence which is faster than the asymptotic one. This has already been observed for fast diffusion equations in Dolbeault and Toscani [2013] with a phenomenon of delay that has been studied in Dolbeault and Toscani [2015a] and by Carrillo and Toscani [2014], by resorting to the concept of Rényi entropy. A remarkable fact is that the inequality is improved by choosing a scale (in practice by imposing a constraint on the second moment) without requesting anything on the first moment, again something that clearly distinguishes the improvements obtained here from what can be guessed by looking at the asymptotic problem as $t \rightarrow \infty$. Details and statements on these consequences for diffusion equations have been collected in Section 5 .

\section{Stability results for the logarithmic Sobolev inequality}

Let $d \mu=\mu d x$ be the normalized Gaussian measure, with $\mu(x)=(2 \pi)^{-d / 2} e^{-|x|^{2} / 2}$, on the Euclidean space $\mathbb{R}^{d}$ with $d \geq 1$. The Gaussian logarithmic Sobolev inequality reads

$$
\int_{\mathbb{R}^{d}}|\nabla u|^{2} d \mu \geq \frac{1}{2} \int_{\mathbb{R}^{d}}|u|^{2} \log |u|^{2} d \mu
$$

for any function $u \in \mathrm{H}^{1}\left(\mathbb{R}^{d}, d \mu\right)$ such that $\int_{\mathbb{R}^{d}}|u|^{2} d \mu=1$. This inequality is equivalent to the Euclidean logarithmic Sobolev inequality in scale invariant form

$$
\frac{d}{2} \log \left(\frac{2}{\pi d e} \int_{\mathbb{R}^{d}}|\nabla w|^{2} d x\right) \geq \int_{\mathbb{R}^{d}}|w|^{2} \log |w|^{2} d x
$$

that can be found in [Weissler, 1978, Theorem 2] in the framework of scalings, but is also the one that can be found in [Stam, 1959, Inequality (2.3)] or in [Carlen, 1991, Inequality (26)]. See Bobkov et al. [2014], Fathi et al. [2014] and Toscani [2014b] for more comments. The equivalence of (1) and (2) is well known but involves some scalings and we will give a short proof below for completeness. Next, let us consider the function

$$
\varphi(t):=\frac{d}{4}\left[\exp \left(\frac{2 t}{d}\right)-1-\frac{2 t}{d}\right] \quad \forall t \in \mathbb{R} .
$$


Our first result is an improvement of (1), based on the comparison of (1) with (2), which combines ideas of Bakry and Ledoux [2006] and Fathi et al. [2014]. It goes as follows.

Proposition 1. With $\varphi$ defined by (3), we have

$$
\begin{array}{r}
\int_{\mathbb{R}^{d}}|\nabla u|^{2} d \mu-\frac{1}{2} \int_{\mathbb{R}^{d}}|u|^{2} \log |u|^{2} d \mu \geq \varphi\left(\int_{\mathbb{R}^{d}}|u|^{2} \log |u|^{2} d \mu\right) \\
\forall u \in \mathrm{H}^{1}\left(\mathbb{R}^{d}, d \mu\right) \quad \text { such that } \int_{\mathbb{R}^{d}}|u|^{2} d \mu=1 \quad \text { and } \quad \int_{\mathbb{R}^{d}}|x|^{2}|u|^{2} d \mu=d
\end{array}
$$

Inequality (4) is an improvement of (1) because $\varphi(t) \geq \frac{t^{2}}{2 d}$ for any $t \in \mathbb{R}$ and, by the Pinsker-CsiszárKullback inequality,

$$
\int_{\mathbb{R}^{d}}|u|^{2} \log |u|^{2} d \mu \geq \frac{1}{4}\left(\left.\int_{\mathbb{R}^{d}}|| u\right|^{2}-1 \mid d \mu\right)^{2} \quad \forall u \in \mathrm{L}^{2}\left(\mathbb{R}^{d}, d \mu\right) \quad \text { such that }\|u\|_{\mathrm{L}^{2}\left(\mathbb{R}^{d}, d \mu\right)}=1 .
$$

See Pinsker [1964], Csiszár [1967], Kullback [1968] for a proof of this inequality.

Proof. To emphasize the role of scalings, let us give a proof of Proposition 1, which follows the strategy of [Bakry and Ledoux, 2006, Proposition 2, p. 694].

As a preliminary step, we recover the scale invariant, Euclidean, version of the logarithmic Sobolev inequality from (1). Let $v:=u \sqrt{\mu}$. We observe that $\int_{\mathbb{R}^{d}}|v|^{2} d x=1$ and $\int_{\mathbb{R}^{d}}|x|^{2}|v|^{2} d x=d$. With one integration by parts, we get that

$$
\int_{\mathbb{R}^{d}}|\nabla v|^{2} d x \geq \frac{1}{2} \int_{\mathbb{R}^{d}}|v|^{2} \log |v|^{2} d x+\frac{d}{4} \log \left(2 \pi e^{2}\right)
$$

which is the standard Euclidean logarithmic Sobolev inequality established in Gross [1975] (also see Federbush [1969] for an earlier related result). This inequality is not invariant under scaling. By considering $w$ such that $v(x)=\lambda^{d / 2} w(\lambda x)$, we get that

$$
\lambda^{2} \int_{\mathbb{R}^{d}}|\nabla w|^{2} d x-\frac{d}{2} \log \lambda \geq \frac{1}{2} \int_{\mathbb{R}^{d}}|w|^{2} \log |w|^{2} d x+\frac{d}{4} \log \left(2 \pi e^{2}\right) .
$$

holds for any $v \in \mathrm{H}^{1}\left(\mathbb{R}^{d}, d \mu\right)$ such that $\|v\|_{\mathrm{L}^{2}\left(\mathbb{R}^{d}\right)}=1$. An optimization on the scaling parameter shows that $4 \lambda^{2} \int_{\mathbb{R}^{d}}|\nabla w|^{2} d x=d$ and establishes the scale invariant form of the logarithmic Sobolev inequality,

$$
\frac{d}{2} \log \left(\frac{2}{\pi d e} \int_{\mathbb{R}^{d}}|\nabla w|^{2} d x\right) \geq \int_{\mathbb{R}^{d}}|w|^{2} \log |w|^{2} d x \quad \forall w \in \mathrm{H}^{1}\left(\mathbb{R}^{d}, d x\right) \quad \text { such that }\|w\|_{\mathrm{L}^{2}\left(\mathbb{R}^{d}\right)}=1,
$$

which is equivalent to (2). This inequality can also be written as

$$
\int_{\mathbb{R}^{d}}|\nabla w|^{2} d x \geq \frac{1}{2} \pi d e \exp \left(\frac{2}{d} \int_{\mathbb{R}^{d}}|w|^{2} \log |w|^{2} d x\right)
$$

If we redefine $u$ such that $w=u \sqrt{\mu}$ and assume that $\|w\|_{\mathrm{L}^{2}\left(\mathbb{R}^{d}\right)}=1, \int_{\mathbb{R}^{d}}|x|^{2} w d x=d$, we have shown that

$$
\int_{\mathbb{R}^{d}}|\nabla u|^{2} d \mu \geq \frac{d}{4}\left[\exp \left(\frac{2}{d} \int_{\mathbb{R}^{d}}|u|^{2} \log |u|^{2} d \mu\right)-1\right] .
$$

Inequality (4) follows by substracting $\frac{1}{2} \int_{\mathbb{R}^{d}}|u|^{2} \log |u|^{2} d \mu$ from both sides of the inequality, which is more or less the idea that has been exploited by Fathi et al. [2014].

Consider a nonnegative function $f \in \mathrm{L}_{2}^{1}\left(\mathbb{R}^{d}\right):=\left\{g \in \mathrm{L}^{1}\left(\mathbb{R}^{d}\right): \int_{\mathbb{R}^{d}}|x|^{2} g d x<\infty\right\}$ and, assuming that $\int_{\mathbb{R}^{d}} f d x>0$, define

$$
M_{f}:=\int_{\mathbb{R}^{d}} f d x, \quad \theta_{f}:=\frac{1}{d} \frac{\int_{\mathbb{R}^{d}}|x|^{2} f d x}{M_{f}} .
$$

Let us define the Gaussian function

$$
\mu_{f}(x):=\frac{M_{f}}{\left(2 \pi \theta_{f}\right)^{d / 2}} e^{-\frac{|x|^{2}}{2 \theta_{f}}} \quad \forall x \in \mathbb{R}^{d} .
$$

We shall denote by $\mathrm{L}_{2}^{1}\left(\mathbb{R}^{d}\right)$ the space of integrable functions on $\mathbb{R}^{d}$ with finite second moment. 
Lemma 2. Assume that $f$ is a nontrivial, nonnegative function in $f \in \mathrm{L}_{2}^{1}\left(\mathbb{R}^{d}\right)$ such that $\nabla \sqrt{f} \in \mathrm{L}^{2}\left(\mathbb{R}^{d}\right)$. With $\theta_{f}, \mu_{f}$ and $\varphi$ defined by (3) and (8), we have

$$
\frac{\theta_{f}}{2} \int_{\mathbb{R}^{d}} \frac{|\nabla f|^{2}}{f} d x-\int_{\mathbb{R}^{d}} f \log f d x-\frac{d}{2} \log \left(2 \pi e^{2} \theta_{f}\right) \int_{\mathbb{R}^{d}} f d x \geq 2 \varphi\left[\int_{\mathbb{R}^{d}} f \log \left(\frac{f}{\mu_{f}}\right) d x\right] .
$$

Proof. Let $v$ be such that $\lambda^{d} f(\lambda x)=|u(x)|^{2} \mu(x)$ with $\lambda^{2}=\theta_{f}, \mu(x)=(2 \pi)^{-d / 2} e^{-|x|^{2} / 2}$, and apply Proposition 1 .

The Gaussian function $\mu_{f}$ is the minimizer of the relative entropy

$$
\mathrm{e}[f \mid \mu]:=\int_{\mathbb{R}^{d}}\left[f \log \left(\frac{f}{\mu}\right)-(f-\mu)\right] d x
$$

w.r.t. all Gaussian functions in

$$
\mathcal{M}:=\left\{\mu(x)=\frac{M}{(2 \pi \theta)^{d / 2}}: e^{-\frac{|x|^{2}}{2 \theta}}, M>0, \theta>0\right\},
$$

that is, we have the identity

$$
\int_{\mathbb{R}^{d}} f \log \left(\frac{f}{\mu_{f}}\right) d x=\mathrm{e}\left[f \mid \mu_{f}\right]=\min \{\mathrm{e}[f \mid \mu]: \mu \in \mathcal{M}\} .
$$

Also notice that $\mu_{f}$ is the minimizer of the relative Fisher information w.r.t. all Gaussian functions of mass $M_{f}$ :

$$
\int_{\mathbb{R}^{d}}\left|\nabla \sqrt{f / \mu_{f}}\right|^{2} d x=\min \left\{\int_{\mathbb{R}^{d}}|\nabla \sqrt{f / \mu}|^{2} d x: \mu(x)=\frac{M_{f}}{(2 \pi \theta)^{d / 2}} e^{-\frac{|x|^{2}}{2 \theta}}, \theta>0\right\} .
$$

Recall that by the Pinsker-Csiszár-Kullback inequality, the r.h.s. in (9) provides an explicit stability result in $\mathrm{L}_{+}^{1}\left(\mathbb{R}^{d}, d x\right)$ that can be written as

$$
\mathrm{e}\left[f \mid \mu_{f}\right] \geq \frac{1}{4 M_{f}}\left\|f-\mu_{f}\right\|_{\mathrm{L}^{1}\left(\mathbb{R}^{d}\right)}^{2} \quad \forall f \in \mathrm{L}_{+}^{1}\left(\mathbb{R}^{d}, d x\right) .
$$

Combined with the observation that $\varphi$ is nondecreasing and $\varphi(t) \geq \frac{t^{2}}{2 d}$ for any $t \in \mathbb{R}$, we have shown the following global stability result.

Corollary 3. Assume that $f$ is a nontrivial, nonnegative function in $f \in \mathrm{L}_{2}^{1}\left(\mathbb{R}^{d}\right)$ such that $\nabla \sqrt{f} \in \mathrm{L}^{2}\left(\mathbb{R}^{d}\right)$. With $\theta_{f}, \mu_{f}$ and $\varphi$ defined by (3) and (8), we have

$$
\begin{aligned}
\frac{\theta_{f}}{2} \int_{\mathbb{R}^{d}} \frac{|\nabla f|^{2}}{f} d x-\int_{\mathbb{R}^{d}} f \log f d x-\frac{d}{2} \log \left(2 \pi e^{2} \theta_{f}\right) & \int_{\mathbb{R}^{d}} f d x \\
& \geq 2 \min _{\mu \in \mathcal{M}} \varphi(\mathrm{e}[f \mid \mu])=2 \varphi\left(\mathrm{e}\left[f \mid \mu_{f}\right]\right) \geq \frac{\left\|f-\mu_{f}\right\|_{\mathrm{L}^{1}\left(\mathbb{R}^{d}\right)}^{4}}{16 M_{f}^{2}}
\end{aligned}
$$

\section{An improved version of the generalized Poincaré inequalities for Gaussian measures}

We consider the inequalities introduced by W. Beckner in [Beckner, 1989, theorem 1]. If $\mu(x)=(2 \pi)^{-d / 2} e^{-|x|^{2} / 2}$, then for any $p \in[1,2)$ we have

$$
\|u\|_{\mathrm{L}^{2}\left(\mathbb{R}^{d}, d \mu\right)}^{2}-\|u\|_{\mathrm{L}^{p}\left(\mathbb{R}^{d}, d \mu\right)}^{2} \leq(2-p)\|\nabla u\|_{\mathrm{L}^{2}\left(\mathbb{R}^{d}, d \mu\right)}^{2} \quad \forall u \in \mathrm{H}^{1}\left(\mathbb{R}^{d}, d \mu\right) .
$$

These inequalities interpolate between the Poincaré inequality ( $p=1$ case) and the logarithmic Sobolev inequality, which is achieved by dividing both sides of the inequality by $(2-p)$ and passing to the limit as $p \rightarrow 2$. Some improvements were obtained already obtained in Arnold and Dolbeault [2005], Arnold et al. [2007], Bartier and Dolbeault [2006]. What we gain here is that the improvement takes place also in the limit case as $p \rightarrow 2$ and is consistent with the results of Proposition 1.

Let us define

$$
\varphi_{p}(x):=\frac{d}{4}\left[(1-x)^{-\frac{2 p}{d(2-p)}}-1\right] \quad \forall x \in[0,1] .
$$


Corollary 4. Assume that $u \in \mathrm{H}^{1}\left(\mathbb{R}^{d}, d \mu\right)$ is such that $\int_{\mathbb{R}^{d}}|u|^{2}|x|^{2} d \mu=d\|u\|_{\mathrm{L}^{2}\left(\mathbb{R}^{d}, d \mu\right)}^{2}$. With the above notation, for any $p \in[1,2)$ we have

$$
\int_{\mathbb{R}^{d}}|\nabla u|^{2} d \mu \geq\|u\|_{\mathrm{L}^{2}\left(\mathbb{R}^{d}, d \mu\right)}^{2} \varphi_{p}\left(\frac{\|u\|_{\mathrm{L}^{2}\left(\mathbb{R}^{d}, d \mu\right)}^{2}-\|u\|_{\mathrm{L}^{p}\left(\mathbb{R}^{d}, d \mu\right)}^{2}}{\|u\|_{\mathrm{L}^{2}\left(\mathbb{R}^{d}, d \mu\right)}^{2}}\right) .
$$

By homogeneity we can assume that $\|u\|_{\mathrm{L}^{2}\left(\mathbb{R}^{d}, d \mu\right)}=1$. The reader is invited to check that

$$
\lim _{p \rightarrow 2} \varphi_{p}\left(1-\|u\|_{\mathrm{L}^{p}\left(\mathbb{R}^{d}, d \mu\right)}^{2}\right)=\frac{d}{4}\left(e^{\frac{2}{d} \mathrm{E}[u]}-1\right) \quad \text { where } \quad \mathrm{E}[u]:=\int_{\mathbb{R}^{d}} \frac{|u|^{2}}{\|u\|_{\mathrm{L}^{2}\left(\mathbb{R}^{d}, d \mu\right)}^{2}} \log \left(\frac{|u|^{2}}{\|u\|_{\mathrm{L}^{2}\left(\mathbb{R}^{d}, d \mu\right)}^{2}}\right) d \mu .
$$

The proof of Corollary 4 is a straightforward consequence of (7) and of the following estimate.

Lemma 5. For any $p \in[1,2)$ and any function $u \in \mathrm{L}^{p} \cap \mathrm{L}^{2}\left(\mathbb{R}^{d}, d \mu\right)$, we have

$$
\frac{\|u\|_{\mathrm{L}^{2}\left(\mathbb{R}^{d}, d \mu\right)}^{2}}{\|u\|_{\mathrm{L}^{p}\left(\mathbb{R}^{d}, d \mu\right)}^{2}} \leq \exp \left(\frac{2-p}{p} \mathrm{E}[u]\right)
$$

and, as a consequence, for any $u \in \mathrm{L}^{p} \cap \mathrm{L}^{2}\left(\mathbb{R}^{d}, d \mu\right)$ such that $\|u\|_{\mathrm{L}^{2}\left(\mathbb{R}^{d}, d \mu\right)}=1$, we obtain

$$
\|u\|_{\mathrm{L}^{2}\left(\mathbb{R}^{d}, d \mu\right)}^{2}-\|u\|_{L^{p}\left(\mathbb{R}^{d}, d \mu\right)}^{2} \leq \frac{2-p}{p} \int_{\mathbb{R}^{d}}|u|^{2} \log |u|^{2} d \mu .
$$

Proof. The proof relies on an idea that can be found in Latała and Oleszkiewicz [2000] and goes as follows. Let us consider the function

$$
k(s):=s \log \left(\int_{\mathbb{R}^{d}} u^{\frac{2}{s}} d \mu\right) .
$$

Derivatives are such that

$$
\begin{aligned}
& \frac{1}{2} k^{\prime}(s)=\log \left(\int_{\mathbb{R}^{d}} u^{\frac{2}{s}} d \mu\right)-\frac{1}{s} \frac{\int_{\mathbb{R}^{d}} u^{\frac{2}{s}} \log u d \mu}{\int_{\mathbb{R}^{d}} u^{\frac{2}{s}} d \mu}, \\
& \frac{s^{3}}{4}\left(\int_{\mathbb{R}^{d}} u^{\frac{2}{s}} d \mu\right)^{2} k^{\prime \prime}(s)=\int_{\mathbb{R}^{d}} u^{\frac{2}{s}} d \mu \int_{\mathbb{R}^{d}} u^{\frac{2}{s}}|\log u|^{2} d \mu-\left(\int_{\mathbb{R}^{d}} \log u u^{\frac{2}{s}} d \mu\right)^{2},
\end{aligned}
$$

hence proving that $k$ is convex by the Cauchy-Schwarz inequality. As a consequence we get that

$$
k^{\prime}(1) \leq \frac{k(s)-k(1)}{s-1} \quad \forall s>1 .
$$

Applied with $s=2 / p$, this proves that

$$
-\int_{\mathbb{R}^{d}}|u|^{2} \log \left(\frac{|u|^{2}}{\|u\|_{\mathrm{L}^{2}\left(\mathbb{R}^{d}, d \mu\right)}^{2}}\right) d \mu \leq \frac{p}{2-p}\|u\|_{\mathrm{L}^{2}\left(\mathbb{R}^{d}, d \mu\right)}^{2} \log \left(\frac{\|u\|_{\mathrm{L}^{p}\left(\mathbb{R}^{d}, d \mu\right)}^{2}}{\|u\|_{\mathrm{L}^{2}\left(\mathbb{R}^{d}, d \mu\right)}^{2}}\right),
$$

from which we deduce the second inequality in (12) after observing that $-\log x \geq 1-x$.

The result of Corollary 4 deserves a comment. As $x \rightarrow 0, \varphi_{p}(x) \sim \frac{p}{2} \frac{x}{2-p}$, so that we do not recover the optimal constant in (10) in the asymptotic regime corresponding to $\|u\|_{\mathrm{L}^{p}\left(\mathbb{R}^{d}, d \mu\right)} /\|u\|_{\mathrm{L}^{2}\left(\mathbb{R}^{d}, d \mu\right)} \rightarrow 1$, that is when $u$ approaches a constant, because of the factor $\frac{p}{2}$. On the other hand, (11) is a strict improvement compared to $(10)$ as soon as $\|u\|_{L^{p}\left(\mathbb{R}^{d}, d \mu\right)}^{2} /\|u\|_{\mathrm{L}^{2}\left(\mathbb{R}^{d}, d \mu\right)}^{2}<x_{\star}(p)$ where $x_{\star}(p)$ is the unique solution to $\varphi_{p}(x)=\frac{x}{2-p}$ in $(0,1)$. Let $\Phi_{p}$ be the function defined by

$$
\Phi_{p}(x)=\varphi_{p}(x) \quad \text { if } \quad x \in\left(0, x_{\star}(p)\right), \quad \Phi_{p}(x)=\frac{x}{2-p} \quad \text { if } \quad x \in\left[x_{\star}(p), 1\right] .
$$


Collecting these estimates of (10) and (11), we can write that

$$
\int_{\mathbb{R}^{d}}|\nabla u|^{2} d \mu \geq\|u\|_{\mathrm{L}^{2}\left(\mathbb{R}^{d}, d \mu\right)}^{2} \Phi_{p}\left(\frac{\|u\|_{\mathrm{L}^{2}\left(\mathbb{R}^{d}, d \mu\right)}^{2}-\|u\|_{\mathrm{L}^{p}\left(\mathbb{R}^{d}, d \mu\right)}^{2}}{\|u\|_{\mathrm{L}^{2}\left(\mathbb{R}^{d}, d \mu\right)}^{2}}\right)
$$

for any function $u \in \mathrm{H}^{1}\left(\mathbb{R}^{d}, d \mu\right)$ such that $\int_{\mathbb{R}^{d}}|u|^{2}|x|^{2} d \mu=d\|u\|_{\mathrm{L}^{2}\left(\mathbb{R}^{d}, d \mu\right)}^{2}$. This is an improvement with respect to (10) because $\Phi_{p}(x) \geq \frac{x}{2-p}$, with a strict inequality if $x<x_{\star}(p)$.

The right hand side in (11) controls the distance to the constants. Indeed, using for instance Hölder's estimates, it is easy to check that

$$
\|u\|_{\mathrm{L}^{2}\left(\mathbb{R}^{d}, d \mu\right)}^{2}-\|u\|_{\mathrm{L}^{p}\left(\mathbb{R}^{d}, d \mu\right)}^{2} \geq\|u\|_{\mathrm{L}^{2}\left(\mathbb{R}^{d}, d \mu\right)}^{2}-\|u\|_{\mathrm{L}^{1}\left(\mathbb{R}^{d}, d \mu\right)}^{2}=\int_{\mathbb{R}^{d}}|u-\bar{u}|^{2} d \mu
$$

with $\bar{u}=\int_{\mathbb{R}^{d}}|u| d \mu$. Sharper estimates based for instance on variants of the Pinsker-Csiszár-Kullback inequality can be found in Cáceres et al. [2002], Bartier et al. [2007].

\section{Stability results for some Gagliardo-Nirenberg inequalities}

\subsection{A first case: $q>1$}

We study the case of Gagliardo-Nirenberg inequalities

$$
\|\nabla w\|_{\mathrm{L}^{2}\left(\mathbb{R}^{d}\right)}^{\vartheta}\|w\|_{\mathrm{L}^{q+1}\left(\mathbb{R}^{d}\right)}^{1-\vartheta} \geq \mathrm{C}_{\mathrm{GN}}\|w\|_{\mathrm{L}^{2 q}\left(\mathbb{R}^{d}\right)}
$$

with $\vartheta=\frac{d}{q} \frac{q-1}{d+2-q(d-2)}$. The value of the optimal constant has been established in Del Pino and Dolbeault [2002] (also see Gunson [1991] for an earlier but partial contribution).

Let us start with some elementary observations on convexity. Consider two positive constants $a$ and $b$. Let us define

$$
\zeta=\frac{b}{a+b}, \quad \kappa=\left(\frac{a}{b}\right)^{\zeta}+\left(\frac{b}{a}\right)^{1-\zeta}=\frac{a+b}{a^{1-\zeta} b^{\zeta}} .
$$

Next let us take three positive numbers, $A, B$, and $C$ such that $A^{\zeta} B^{1-\zeta} \geq C$ and consider the function

$$
h(\lambda)=\lambda^{a} A+\lambda^{-b} B-\kappa C .
$$

The function $h$ reaches its minimum at $\lambda=\lambda_{*}:=\left(\frac{b B}{a A}\right)^{\frac{1}{a+b}}$ and it is straightforward to check that

$$
h(1) \geq \inf _{\lambda>0} h(\lambda)=h\left(\lambda_{*}\right)=\kappa\left(A^{\zeta} B^{1-\zeta}-C\right)
$$

This computation determines the choice of $\kappa$. Using the assumption $A^{\zeta} B^{1-\zeta} \geq C$, we get the estimate

$$
A+B-\kappa C \geq C^{\frac{1}{\zeta}} B^{1-\frac{1}{\zeta}}+B-\kappa C=\varphi\left(B_{*}-B\right)
$$

where

$$
B_{*}:=C\left(\frac{1-\zeta}{\zeta}\right)^{\zeta}
$$

and

$$
\varphi(s):=C^{\frac{1}{\zeta}}\left[\left(B_{*}-s\right)^{1-\frac{1}{\zeta}}-B_{*}^{1-\frac{1}{\zeta}}\right]-s .
$$

Indeed, $\zeta=\frac{b}{a+b}$ leads to the identity

$$
\kappa C=B_{*}+C^{\frac{1}{\zeta}} B_{*}^{1-\frac{1}{\zeta}} .
$$

Note that $\varphi$ is a nonnegative strictly convex function such that $\varphi(0)=0$ and $\varphi^{\prime \prime}(s)>\varphi^{\prime \prime}(0)=\frac{1-\zeta}{\zeta^{2}} C^{\frac{1}{\zeta}} B_{*}^{-1-\frac{1}{\zeta}}$ for any $s \in\left(0, B_{*}\right)$. 
We apply these preliminary computations with

$$
\begin{aligned}
& a=\frac{d}{q}-(d-2), \quad b=d \frac{q-1}{2 q} \\
& A=\frac{1}{4}\left(q^{2}-1\right) \int_{\mathbb{R}^{d}}|\nabla w|^{2} d x, \quad B=\beta \int_{\mathbb{R}^{d}}|w|^{q+1} d x, \quad \beta=\frac{2 q}{q-1}-d \\
& C=\left(\frac{1}{4}\left(q^{2}-1\right)\right)^{\zeta} \beta^{1-\zeta}\left(\mathrm{C}_{\mathrm{GN}}\|w\|_{\mathrm{L}^{2 q}\left(\mathbb{R}^{d}\right)}\right)^{\alpha}, \quad \alpha=q+1-\zeta(q-1)
\end{aligned}
$$

for any $q \in\left(1, \frac{d}{d-2}\right)$. With $\mathcal{K}:=\left(\frac{1}{4}\left(q^{2}-1\right)\right)^{\zeta} \beta^{1-\zeta} \kappa$, the functional

$$
\mathrm{J}[w]:=\frac{1}{4}\left(q^{2}-1\right) \int_{\mathbb{R}^{d}}|\nabla w|^{2} d x+\beta \int_{\mathbb{R}^{d}}|w|^{q+1} d x-\mathcal{K} \mathrm{C}_{\mathrm{GN}}^{\alpha}\left(\int_{\mathbb{R}^{d}}|w|^{2 q} d x\right)^{\frac{\alpha}{2 q}}
$$

is nonnegative and achieves its minimum at $w_{*}(x)=\left(1+|x|^{2}\right)^{\frac{1}{1-q}}$. Hence we have that

$$
\mathrm{J}[w] \geq \mathrm{J}\left[w_{*}\right]=0
$$

and this inequality is equivalent to (14), after an optimization under scaling. Notice that $\vartheta=2 \zeta / \alpha$.

Theorem 6. With the above notations and $\varphi$ given by (15), we have

$$
\mathrm{J}[w] \geq \varphi\left[\beta\left(\int_{\mathbb{R}^{d}}\left|w_{*}\right|^{q+1} d x-\int_{\mathbb{R}^{d}}|w|^{q+1} d x\right)\right]
$$

for any $w \in \mathrm{L}^{q+1}\left(\mathbb{R}^{d}\right)$ such that $\int_{\mathbb{R}^{d}}|\nabla w|^{2} d x<\infty$ and $\int_{\mathbb{R}^{d}}|w|^{2 q}|x|^{2} d x=\int_{\mathbb{R}^{d}} w_{*}^{2 q}|x|^{2} d x$.

Proof. The reader is invited to check that, with the above notations,

$$
B_{*}-B=\beta\left(\int_{\mathbb{R}^{d}}\left|w_{*}\right|^{q+1} d x-\int_{\mathbb{R}^{d}}|w|^{q+1} d x\right) .
$$

As a last remark in this section, let us observe that the logarithmic Sobolev inequality appears as a limit case of the entropy - entropy production inequality, and that (2) is also obtained by taking the limit as $q \rightarrow 1$ in Gagliardo-Nirenberg inequalities in (14): see Del Pino and Dolbeault [2002] for details. Also, when $d \geq 2$, the convexity of $\varphi$ is lost as $q \rightarrow \frac{d}{d-2}$, which corresponds to Sobolev's inequality. This shows the consistancy of our method.

\subsection{A second case: $q<1$}

Now we study the case of Gagliardo-Nirenberg inequalities

$$
\|\nabla w\|_{\mathrm{L}^{2}\left(\mathbb{R}^{d}\right)}^{\vartheta}\|w\|_{\mathrm{L}^{2 q}\left(\mathbb{R}^{d}\right)}^{1-\vartheta} \geq \mathrm{C}_{\mathrm{GN}}\|w\|_{\mathrm{L}^{q+1}\left(\mathbb{R}^{d}\right)}
$$

with $q=\frac{1}{2 p-1}<1, \vartheta=\frac{d}{1+q} \frac{1-q}{d-q(d-2)}$ and we denote by $\|w\|_{L^{2 q}\left(\mathbb{R}^{d}\right)}$ the quantity $\left(\int_{\mathbb{R}^{d}}|w|^{2 q} d x\right)^{\frac{1}{2 q}}$ for any $q \in(0,1)$, even for $q<1 / 2$ (in that case, it is only a semi-norm).

Our elementary estimates have to be adapted. Consider two positive constants $a$ and $b$, with $a>b$. Let us define

$$
\eta=\frac{b}{a-b}, \quad \kappa=\left(\frac{b}{a}\right)^{\eta}-\left(\frac{b}{a}\right)^{1+\eta}=\frac{a-b}{b^{-\eta} a^{1+\eta}} .
$$

Next let us take three positive numbers, $A, B$, and $C$ such that $A^{-\eta} B^{1+\eta} \leq C$ and consider the function

$$
h(\lambda)=\lambda^{a} A-\lambda^{b} B+\kappa C .
$$

The function $h$ reaches its minimum at $\lambda=\lambda_{*}:=\left(\frac{b B}{a A}\right)^{\frac{1}{a-b}}$ and it is straightforward to check that

$$
h(\lambda) \geq h\left(\lambda_{*}\right)=\kappa\left(C-A^{-\eta} B^{1+\eta}\right) .
$$


Using the assumption $A^{-\eta} B^{1+\eta} \leq C$, we get the estimate

$$
A-B+\kappa C \geq C^{-\frac{1}{\eta}} B^{1+\frac{1}{\eta}}-B+\kappa C=\varphi\left(B-B_{*}\right)
$$

where

$$
B_{*}:=C\left(\frac{\eta}{1+\eta}\right)^{\eta}
$$

and

$$
\varphi(s)=C^{-\frac{1}{\eta}}\left[\left(B_{*}+s\right)^{1+\frac{1}{\eta}}-B_{*}^{1+\frac{1}{\eta}}\right]-s
$$

is a nonnegative strictly convex function such that $\varphi(0)=0$ and $\varphi^{\prime \prime}(s)>\varphi^{\prime \prime}(0)>0$ for any $s>0$.

We apply these preliminary computations with

$$
\begin{aligned}
& a=\frac{d}{q}-(d-2), \quad b=d \frac{1-q}{2 q} \\
& A=\frac{1}{4}\left(q^{2}-1\right) \int_{\mathbb{R}^{d}}|\nabla w|^{2} d x, \quad B=\beta \int_{\mathbb{R}^{d}}|w|^{q+1} d x, \quad \beta=\frac{2 q}{1-q}+d \\
& C=\left(\frac{1}{4}\left(q^{2}-1\right)\right)^{-\eta} \beta^{1+\eta}\left(\mathrm{C}_{\mathrm{GN}}\right)^{-(q+1)(1+\eta)}\|w\|_{\mathrm{L}^{2 q}\left(\mathbb{R}^{d}\right)}^{\alpha}, \quad \alpha=q+1+\eta(q-1)
\end{aligned}
$$

for any $q \in(0,1)$. With $\mathcal{K}:=\left(\frac{1}{4}\left(q^{2}-1\right)\right)^{-\eta} \beta^{1+\eta} \kappa$, the functional

$$
\mathrm{J}[w]:=\frac{1}{4}\left(q^{2}-1\right) \int_{\mathbb{R}^{d}}|\nabla w|^{2} d x-\beta \int_{\mathbb{R}^{d}}|w|^{q+1} d x+\mathcal{K}\left(\mathrm{C}_{\mathrm{GN}}\right)^{-(q+1)(1+\eta)}\left(\int_{\mathbb{R}^{d}}|w|^{2 q} d x\right)^{\frac{\alpha}{2 q}}
$$

is nonnegative and achieves its minimum at $w_{*}(x)=\left(1-|x|^{2}\right)_{+}^{\frac{1}{q-1}}$. Hence we have that

$$
\mathrm{J}[w] \geq \mathrm{J}\left[w_{*}\right]=0
$$

and this inequality is equivalent to (17), after an optimization under scaling. Notice that $\vartheta=\frac{2 \eta}{(q+1)(1+\eta)}$.

Theorem 7. With the above notations and $\varphi$ given by (18), we have

$$
\mathrm{J}[w] \geq \varphi\left[\beta\left(\int_{\mathbb{R}^{d}}|w|^{q+1} d x-\int_{\mathbb{R}^{d}}\left|w_{*}\right|^{q+1} d x\right)\right] \quad \forall w \in \mathrm{L}^{q+1}\left(\mathbb{R}^{d}\right) \quad \text { such that } \int_{\mathbb{R}^{d}}|\nabla w|^{2} d x<\infty .
$$

Proof. The proof is similar to the proof of Theorem 6 except that the roles of $\int_{\mathbb{R}^{d}}\left|w_{*}\right|^{q+1} d x$ and $\int_{\mathbb{R}^{d}}\left|w_{*}\right|^{2 q} d x$ are exchanged.

\section{Some consequences for diffusion equations}

\subsection{Linear case: the Ornstein-Uhlenbeck equation}

Let us consider the Ornstein-Uhlenbeck equation (or backward Kolmogorov equation)

$$
\frac{\partial f}{\partial t}=\Delta f-x \cdot \nabla f
$$

with initial datum $f_{0} \in \mathrm{L}_{+}^{1}\left(\mathbb{R}^{d},\left(1+|x|^{2}\right) d \mu\right.$ and define the entropy as

$$
\mathcal{E}[f]:=\int_{\mathbb{R}^{d}} f \log f d \mu .
$$

Using (1), a standard computation shows that a solution $f=(t, \cdot)$ to $(20)$ satisfies

$$
\frac{d}{d t} \mathcal{E}[f]=-4 \int_{\mathbb{R}^{d}}|\nabla \sqrt{f}|^{2} d \mu \leq-2 \mathcal{E}[f],
$$

thus proving that

$$
\mathcal{E}[f(t, \cdot)] \leq \mathcal{E}\left[f_{0}\right] e^{-2 t} \quad \forall t \geq 0 .
$$

It is well known that $M=\int_{\mathbb{R}^{d}} f(t, \cdot) d \mu$ does not depend on $t \geq 0$. Since the second moment evolves according to

$$
\frac{d}{d t} \int_{\mathbb{R}^{d}} f|x|^{2} d \mu=2 \int_{\mathbb{R}^{d}} f\left(d-|x|^{2}\right) d \mu,
$$

if we assume that $\int_{\mathbb{R}^{d}} f_{0}|x|^{2} d \mu=d M$, then we get also that $\int_{\mathbb{R}^{d}} f(t, \cdot)|x|^{2} d \mu=d M$ for any $t \geq 0$. 
Theorem 8. Let $d \geq 1$ and consider a nonnegative solution to (20) with initial datum $f_{0}$ such that $\mathcal{E}\left[f_{0}\right]$ is finite and $\int_{\mathbb{R}^{d}} f_{0}|x|^{2} d \mu=d \int_{\mathbb{R}^{d}} f_{0} d \mu$. Then we have

$$
\mathcal{E}[f(t, \cdot)] \leq-\frac{d}{2} \log \left[1-\left(1-e^{-\frac{2}{d} \mathcal{E}\left[f_{0}\right]}\right) e^{-2 t}\right] \quad \forall t \geq 0 .
$$

Proof. The proof relies on the estimate

$$
\frac{d}{d t} \mathcal{E}[f]=-d \int_{\mathbb{R}^{d}}|\nabla \sqrt{f}|^{2} d \mu \geq \frac{d}{4}\left[\exp \left(\frac{2}{d} \mathcal{E}[f]\right)-1\right]
$$

according to (7).

Let us conclude this section on the Ornstein-Uhlenbeck equation with some remarks.

(i) The estimate (22) is better than (21): if we let $x=e^{-2 t}$ and $a=e^{-\frac{2}{d} \mathcal{E}\left[f_{0}\right]}$, then

$$
-\frac{d}{2} \log \left[1-\left(1-e^{-\frac{2}{d} \mathcal{E}\left[f_{0}\right]}\right) e^{-2 t}\right] \leq \mathcal{E}\left[f_{0}\right] e^{-2 t}
$$

for any $t \geq 0$ is equivalent to prove that $h(x)=1-a^{x}-(1-a) x$ is nonnegative for any $a \geq 0$ and any $x \in(0,1)$. This is indeed the case because $h(0)=h(1)=0$ and $h^{\prime \prime}(x)=-a^{x}(\log x)^{2}<0$.

The improvement degenerates as $\mathcal{E}\left[f_{0}\right] \rightarrow 0_{+}$. Indeed, we may observe that, for a given $t \geq 0$,

$$
-\frac{d}{2} \log \left[1-\left(1-e^{-\frac{2}{d} \mathcal{E}_{0}}\right) e^{-2 t}\right] \sim \mathcal{E}_{0} e^{-2 t} \quad \text { as } \quad \mathcal{E}_{0} \rightarrow 0_{+} .
$$

(ii) Similar results can be obtained using the generalized Poincaré inequalities for Gaussian measures of Section 3. With $q=2 / p$, we get that

$$
\frac{d}{d t} \int_{\mathbb{R}^{d}} \frac{f^{q}-M^{q}}{q-1} d \mu=-\frac{4}{q} \int_{\mathbb{R}^{d}}\left|\nabla f^{q / 2}\right|^{2} d \mu \leq \Psi_{p}\left(\int_{\mathbb{R}^{d}} \frac{f^{q}-M^{q}}{q-1} d \mu\right)
$$

with $\Psi_{p}(t):=\max \left\{2, \frac{2 p}{2-p} \varphi_{p}\left(\frac{2-p}{p} t\right)\right\}=\frac{2 p}{2-p} \Phi_{p}\left(\frac{2-p}{p} t\right)$ with $\Phi_{p}$ as in (13), and thus get an improvement of the standard estimate

$$
\int_{\mathbb{R}^{d}} \frac{f^{q}-M^{q}}{q-1} d \mu \leq \int_{\mathbb{R}^{d}} \frac{f_{0}^{q}-M^{q}}{q-1} d \mu e^{-2 t} \quad \forall t \geq 0
$$

if $\int_{\mathbb{R}^{d}} \frac{f_{0}^{q}-M^{q}}{q-1} d \mu<\frac{p}{2-p} x_{\star}(p)$.

(iii) None of the above improvements requires that $\int_{\mathbb{R}^{d}} f x d \mu=0$. If this condition is added at $t=0$, it is preserved by the flow corresponding to (20) and spectral methods allows to prove that

$$
\int_{\mathbb{R}^{d}} \frac{f^{q}-M^{q}}{q-1} d \mu \leq \int_{\mathbb{R}^{d}} \frac{f_{0}^{q}-M^{q}}{q-1} d \mu e^{-2 \lambda t} \quad \forall t \geq 0
$$

where $\lambda$ is the best constant in the inequality

$$
\|u\|_{\mathrm{L}^{2}\left(\mathbb{R}^{d}, d \mu\right)}^{2}-\|u\|_{\mathrm{L}^{p}\left(\mathbb{R}^{d}, d \mu\right)}^{2} \leq \frac{1}{\lambda}\|\nabla u\|_{\mathrm{L}^{2}\left(\mathbb{R}^{d}, d \mu\right)}^{2}
$$

for any $u \in \mathrm{H}^{1}\left(\mathbb{R}^{d}, d \mu\right)$ such that $\int_{\mathbb{R}^{d}} u\left(1, x,|x|^{2}-d\right) d \mu=(0,0,0)$. According to [Arnold et al., 2007, Theorem 2.4], we know that $\lambda \geq \frac{\lambda_{3}}{1-(p-1)^{\lambda_{3} / \lambda_{1}}}$ where $\left(\lambda_{i}\right)_{i \geq 0}$ are the eigenvalues of the Ornstein-Uhlenbeck operator $-\Delta+x \cdot \nabla$. Standard results on the harmonic oscillator allow us to prove that $\lambda_{i}=i$, where the eigenspaces associated with $i=0, i=1$ and $i=2$ are generated respectively by the constants, $x_{i}$ with $i=1,2, \ldots d$ and $|x|^{2}-d$. 


\subsection{Nonlinear case: the fast diffusion equation}

The inequality $\mathrm{J}[w] \geq 0$ in Section 4.1 is also known as the entropy - entropy-production inequality for the fast diffusion equation, as was shown in Del Pino and Dolbeault [2002]. Also see Arnold et al. [2004] for a review on these methods. Here are some details. Let us consider the free energy

$$
\mathcal{F}[v]:=\frac{1}{p-1} \int_{\mathbb{R}^{d}}\left(v^{p}-\mathfrak{B}^{p}-p \mathfrak{B}^{p-1}(v-\mathfrak{B})\right) d x
$$

where the Barenblatt profile $\mathfrak{B}$ is defined by

$$
\mathfrak{B}(x)=\left(1+|x|^{2}\right)^{\frac{1}{p-1}} \quad \forall x \in \mathbb{R}^{d} .
$$

and has mass

$$
M_{*}:=\int_{\mathbb{R}^{d}} \mathfrak{B} d x=\pi^{\frac{d}{2}} \frac{\Gamma\left(\frac{1}{1-p}-\frac{d}{2}\right)}{\Gamma\left(\frac{1}{1-p}\right)} .
$$

Next we can define the generalized Fisher information by

$$
\mathcal{I}[v]:=\frac{p}{1-p} \int_{\mathbb{R}^{d}} v\left|\nabla v^{p-1}-2 x\right|^{2} d x
$$

and consider the deficit functional

$$
\mathcal{J}[v]:=\mathcal{I}[v]-4 \mathcal{F}[v]
$$

We may also define the temperature as

$$
\Theta[v]:=\frac{1}{d} \frac{\int_{\mathbb{R}^{d}}|x|^{2} v d x}{\int_{\mathbb{R}^{d}} v d x} .
$$

It turns out that $\mathcal{J}[v]=\mathrm{J}[w]$ if $v^{p-\frac{1}{2}}=w$ and $q=\frac{1}{2 p-1}$. Notice that $q \in\left(1, \frac{d}{d-2}\right)$ is equivalent to $p \in\left(p_{1}, 1\right)$ with $p_{1}:=\frac{d-1}{d}$. Recall that $\beta=\frac{2 q}{q-1}-d=\frac{1}{1-p}-d$. If $\Theta[v]=\Theta[\mathfrak{B}]$, we observe that $\mathcal{F}[v]:=\frac{1}{p-1} \int_{\mathbb{R}^{d}}\left(v^{p}-\mathfrak{B}^{p}\right) d x$ because $\int_{\mathbb{R}^{d}} \mathfrak{B}^{p-1}(v-\mathfrak{B}) d x=0$. Theorem 6 can be rephrased in terms of $v$ as follows.

Corollary 9. Let $p \in\left(p_{1}, 1\right)$. Assume that $v$ is a nonnegative function in $\mathrm{L}^{1}\left(\mathbb{R}^{d}\right)$ is such that $v^{p}$ and $v|x|^{2}$ are both in $\mathrm{L}^{1}\left(\mathbb{R}^{d}\right)$, and $\nabla v$ is in $\mathrm{L}^{2}\left(\mathbb{R}^{d}\right)$. With the above notations and $\varphi$ defined by (15) we have

$$
\mathcal{J}[v] \geq \varphi(\beta(1-p) \mathcal{F}[v]) \quad \text { if } \quad \int_{\mathbb{R}^{d}} v d x=M_{*} \quad \text { and } \quad \Theta[v]=\Theta[\mathfrak{B}]
$$

Since $\varphi^{\prime \prime}(s) \geq \varphi^{\prime \prime}(0)$ for any admissible $s \geq 0$, we have in particular that

$$
\mathcal{J}[v] \geq \kappa(\mathcal{F}[v])^{2}
$$

under the assumptions of Corollary 9 , with $\kappa=\frac{1}{2} \beta^{2}(1-p)^{2} \varphi^{\prime \prime}(0)$. Hence Corollary 9 allows to recover [Dolbeault and Toscani, 2013, Theorem 8] with a much simpler proof.

In a second step, we can get rid of the constraints on the mass and on the second moment. Let us define

$$
\Theta_{*}:=\Theta[\mathfrak{B}] .
$$

If we write that

$$
u(x)=\lambda^{1+\alpha d} \sigma^{-\frac{d}{2}} v\left(\lambda^{\alpha} x / \sqrt{\sigma}\right) \quad \text { with } \quad \lambda=\frac{M}{M_{*}}
$$

for some $\alpha \in \mathbb{R}$ to be determined later and choose $\sigma>0$ such that

$$
\sigma=\sigma[u]:=\lambda^{-(1+2 \alpha)} \frac{\int_{\mathbb{R}^{d}} u|x|^{2} d x}{\int_{\mathbb{R}^{d}} \mathfrak{B}|x|^{2} d x},
$$

then we can define the corresponding Barenblatt profile by

$$
\mathfrak{B}_{M, \sigma}(x)=\lambda^{1+\alpha d} \sigma^{-\frac{d}{2}} \mathfrak{B}\left(\lambda^{\alpha} x / \sqrt{\sigma}\right) \quad \forall x \in \mathbb{R}^{d},
$$


and the relative entropy and the relative Fisher information respectively by

$$
\mathcal{F}_{M, \sigma}[u]:=\frac{1}{p-1} \int_{\mathbb{R}^{d}}\left(u^{p}-\mathfrak{B}_{M, \sigma}^{p}-p \mathfrak{B}_{M, \sigma}^{p-1}\left(u-\mathfrak{B}_{M, \sigma}\right)\right) d x
$$

and, with $p_{c}:=\frac{d-2}{d}$,

$$
\mathcal{I}_{M, \sigma}[u]:=\frac{p}{1-p} \sigma^{\frac{d}{2}\left(p-p_{c}\right)} \int_{\mathbb{R}^{d}} u\left|\nabla u^{p-1}-\nabla \mathfrak{B}_{M, \sigma}^{p-1}\right|^{2} d x
$$

The Barenblatt profile $\mathfrak{B}_{M, 1}(x)$ takes the form $\left(C_{M}+|x|^{2}\right)^{1 /(p-1)}$ for some positive constant $C_{M}=\lambda^{\frac{1+\alpha d}{p-1}}$ if and only if

$$
\alpha=\frac{1-p}{2-d(1-p)} .
$$

With this choice, the functionals $\mathcal{F}_{M, \sigma}[u]$ and $\mathcal{I}_{M, \sigma}[u]$ have the same scaling properties, so that we get

$$
\mathcal{J}_{M, \sigma}[u]:=\mathcal{I}_{M, \sigma}[u]-4 \mathcal{F}_{M, \sigma}[u]=\lambda^{\frac{2 p-d(1-p)}{2-d(1-p)}} \sigma^{\frac{d}{2}(1-p)} \mathcal{J}[v]
$$

By expressing the values of $\lambda$ and $\sigma$ in terms of $M$ and $\Theta[u]$, we have that

$$
\begin{gathered}
\lambda=\frac{M}{M_{*}} \text { and } \sigma=\sigma[u]=\left(\frac{M_{*}}{M}\right)^{\frac{2(2-p)-d(1-p)}{2-d(1-p)}} \frac{\Theta[u]}{\Theta_{*}} \\
\lambda^{\frac{2 p-d(1-p)}{2-d(1-p)}} \sigma^{\frac{d}{2}(1-p)}=\mathrm{h}(M, \Theta[u]) \quad \text { with } \mathrm{h}(M, \Theta):=\left(\frac{M}{M_{*}}\right)^{\frac{d^{2}(1-p)^{2}-2 d\left(p^{2}-4 p+3\right)+4 p}{2[2-d(1-p)]}}\left(\frac{\Theta}{\Theta_{*}}\right)^{\frac{d}{2}(1-p)}
\end{gathered}
$$

We can now rephrase Corollary 9 for a general function $u$ as follows.

Corollary 10. Let $p \in\left(p_{1}, 1\right)$. Assume that $u$ is a nonnegative function in $\mathrm{L}^{1}\left(\mathbb{R}^{d}\right)$ is such that $u^{p}$ and $u|x|^{2}$ are both in $\mathrm{L}^{1}\left(\mathbb{R}^{d}\right)$, and $\nabla u$ is in $\mathrm{L}^{2}\left(\mathbb{R}^{d}\right)$. With the same notations as in Corollary 9 and $\varphi$ defined by $(15)$ we have

$$
\mathcal{J}[u]_{M, \sigma} \geq \mathrm{h}(M, \Theta[u]) \varphi\left(\beta(1-p) \frac{\mathcal{F}_{M, \sigma}[u]}{\mathrm{h}(M, \Theta[u])}\right) \quad \text { with } \quad \sigma=\sigma[u]
$$

The choice $\sigma=\sigma[u]$ is remarkable because

$$
\mathcal{F}_{M, \sigma[u]}[u]=\inf _{\sigma>0} \mathcal{F}_{M, \sigma}[u]
$$

so that $\mathfrak{B}_{M, \sigma[u]}$ is the best matching Barenblatt profile, among all Barenblatt profiles with mass $M$ and characteristic scale $\sigma>0$, when measured in relative entropy. See Dolbeault and Toscani [2011, 2013] for more details.

One of the interests of the statement of Corollary 10 is that the free energy $\mathcal{F}_{M, \sigma[u]}[u]$ is an explicit distance to the manifold of the optimal functions for the Gagliardo-Nirenberg inequalities. We have indeed the following Csiszár-Kullback inequality.

Theorem 11. [Dolbeault and Toscani, 2013, Theorem 4] Let $d \geq 1, p \in(d /(d+2), 1)$ and assume that $u$ is a non-negative function in $\mathrm{L}^{1}\left(\mathbb{R}^{d}\right)$ such that $u^{p}$ and $x \mapsto|x|^{2} u$ are both integrable on $\mathbb{R}^{d}$. If $\|u\|_{\mathrm{L}^{1}\left(\mathbb{R}^{d}\right)}=M$, then

$$
\mathcal{F}_{M, \sigma[u]}[u] \geq \frac{p \sigma[u]^{\frac{d}{2}(1-p)}}{8 \int_{\mathbb{R}^{d}} \mathfrak{B}_{M, 1}^{p} d x}\left(C_{M}\left\|u-\mathfrak{B}_{M, \sigma[u]}\right\|_{L^{1}\left(\mathbb{R}^{d}\right)}+\frac{1}{\sigma[u]} \int_{\mathbb{R}^{d}}|x|^{2}\left|u-\mathfrak{B}_{M, \sigma[u]}\right| d x\right)^{2}
$$

The fast diffusion equation written in self-similar variables is

$$
\frac{\partial u}{\partial t}+\nabla\left[v \cdot\left(\sigma^{\frac{d}{2}\left(p-p_{c}\right)} \nabla v^{p-1}-2 x\right)\right]=0
$$


and it is equivalent to the fast diffusion equation $\frac{\partial v}{\partial t}=\Delta v^{p}$ up to a rescaling: see for instance Del Pino and Dolbeault [2002], Blanchet et al. [2009] when $\sigma$ is taken constant and Dolbeault and Toscani [2011, 2013, 2015b] when $\sigma=\sigma[u(t, \cdot)]$ depends on $t$. By using Corollary 10, we get that

$$
\frac{d}{d t} \mathcal{F}_{M, \sigma[u(t, \cdot)]}[u(t, \cdot)]=-\mathcal{I}_{M, \sigma[u(t, \cdot)]}[u(t, \cdot)]
$$

while a direct computation shows that

$$
\frac{d}{d t} \sigma[u(t, \cdot)]=-\frac{2(1-p)^{2}}{p M \Theta\left[\mathfrak{B}_{M, 1}\right]} \sigma[u(t, \cdot)]^{\frac{d}{2}\left(p-p_{c}\right)} \mathcal{F}_{M, \sigma[u(t, \cdot)]}[u(t, \cdot)] \leq 0 .
$$

Altogether this establishes a faster convergence rate of the solutions towards the Barenblatt profiles than one would get using the entropy - entropy production inequality $\mathcal{J}_{M, \sigma[u]}[u] \geq 0$, and even a better rate that the one found in Dolbeault and Toscani $[2013,2015 \mathrm{~b}]$ because $\varphi^{\prime \prime}(s)>\varphi^{\prime \prime}(0)$. Details are out of the scope of this paper and a simplified method will appear in Dolbeault and Toscani [2015b].

\subsection{Nonlinear case: the porous medium equation}

Now we turn our attention to the inequality $\mathrm{J}[w] \geq 0$ in Section 4.2 which was also studied in Del Pino and Dolbeault [2002]. With $p>1$, we may consider the free energy

$$
\mathcal{F}[v]:=\frac{1}{p-1} \int_{\mathbb{R}^{d}}\left(v^{p}-\mathfrak{B}^{p}+p|x|^{2}(v-\mathfrak{B})\right) d x
$$

for any nonnegative function $v$ such that $\int_{\mathbb{R}^{d}} v d x=\int_{\mathbb{R}^{d}} \mathfrak{B} d x$, where the Barenblatt profile $\mathfrak{B}$ is now defined by

$$
\mathfrak{B}(x)=\left(1-|x|^{2}\right)_{+}^{\frac{1}{p-1}} \quad \forall x \in \mathbb{R}^{d} .
$$

and has mass

$$
M_{*}:=\pi^{\frac{d}{2}} \frac{\Gamma\left(\frac{p}{p-1}\right)}{\Gamma\left(\frac{p}{p-1}+\frac{d}{2}\right)} .
$$

Next we can define the generalized Fisher information by

$$
\mathcal{I}[v]:=\frac{p}{p-1} \int_{\mathbb{R}^{d}} v\left|\nabla v^{p-1}-2 x\right|^{2} d x
$$

and consider the deficit functional

$$
\mathcal{J}[v]:=\mathcal{I}[v]-4 \mathcal{F}[v]
$$

As before, we may also define the temperature as

$$
\Theta[v]:=\frac{1}{d} \frac{\int_{\mathbb{R}^{d}}|x|^{2} v d x}{\int_{\mathbb{R}^{d}} v d x}
$$

It turns out that $\mathcal{J}[v]=\mathrm{J}[w]$ if $v^{p-\frac{1}{2}}=w$ and $q=\frac{1}{2 p-1}$. Notice that $q \in(0,1)$ is equivalent to $p \in(1, \infty)$. Recall that $\beta=\frac{2 q}{1-q}+d=\frac{1}{p-1}+d$. If $\Theta[v]=\Theta[\mathfrak{B}]$, we observe that $\mathcal{F}[v]:=\frac{1}{p-1} \int_{\mathbb{R}^{d}}\left(v^{p}-\mathfrak{B}^{p}\right) d x$. Theorem 7 can be rephrased in terms of $v$ as follows.

Corollary 12. Let $p \in(1,+\infty)$. Assume that $v$ is a nonnegative function in $\mathrm{L}^{1}\left(\mathbb{R}^{d}\right)$ is such that $v^{p}$ and $v|x|^{2}$ are both in $\mathrm{L}^{1}\left(\mathbb{R}^{d}\right)$, and $\nabla v$ is in $\mathrm{L}^{2}\left(\mathbb{R}^{d}\right)$. With the above notations and $\varphi$ defined by (18) we have

$$
\mathcal{J}[v] \geq \varphi(\beta(p-1) \mathcal{F}[v]) \quad \text { if } \quad \int_{\mathbb{R}^{d}} v d x=M_{*} \quad \text { and } \quad \Theta[v]=\Theta[\mathfrak{B}]
$$


Since $\varphi^{\prime \prime}(s) \geq \varphi^{\prime \prime}(0)$ for any admissible $s \geq 0$, we have in particular that

$$
\mathcal{J}[v] \geq \kappa(\mathcal{F}[v])^{2}
$$

under the assumptions of Corollary 12, with $\kappa=\frac{1}{2} \beta^{2}(1-p)^{2} \varphi^{\prime \prime}(0)$. The result of Corollary 12 is new.

In a second step, we can get rid of the constraints on the mass and on the second moment. Let us define

$$
\Theta_{*}:=\Theta[\mathfrak{B}]
$$

If we write that

$$
u(x)=\lambda^{1+\alpha d} \sigma^{-\frac{d}{2}} v\left(\lambda^{\alpha} x / \sqrt{\sigma}\right) \quad \text { with } \quad \lambda=\frac{M}{M_{*}}
$$

for some $\alpha \in \mathbb{R}$ to be determined later and choose $\sigma>0$ such that

$$
\sigma=\sigma[u]:=\lambda^{-(1+2 \alpha)} \frac{\int_{\mathbb{R}^{d}} u|x|^{2} d x}{\int_{\mathbb{R}^{d}} \mathfrak{B}|x|^{2} d x},
$$

then we can define the corresponding Barenblatt profile by

$$
\mathfrak{B}_{M, \sigma}(x)=\lambda^{1+\alpha d} \sigma^{-\frac{d}{2}} \mathfrak{B}\left(\lambda^{\alpha} x / \sqrt{\sigma}\right) \quad \forall x \in \mathbb{R}^{d},
$$

and the relative entropy and the relative Fisher information respectively by

$$
\mathcal{F}_{M, \sigma}[u]:=\frac{1}{p-1} \int_{\mathbb{R}^{d}}\left(u^{p}-\mathfrak{B}_{M, \sigma}^{p}+p \sigma^{-\frac{d}{2}\left(p-p_{c}\right)}|x|^{2}\left(u-\mathfrak{B}_{M, \sigma}\right)\right) d x
$$

and

$$
\mathcal{I}_{M, \sigma}[u]:=\frac{p}{p-1} \sigma^{\frac{d}{2}\left(p-p_{c}\right)} \int_{\mathbb{R}^{d}} u\left|\nabla u^{p-1}-2 x \sigma^{-\frac{d}{2}\left(p-p_{c}\right)}\right|^{2} d x .
$$

where $p_{c}=\frac{d-2}{d}$. The Barenblatt profile $\mathfrak{B}_{M, 1}(x)$ takes the form $\left(C_{M}-|x|^{2}\right)_{+}^{1 /(p-1)}$ for some positive constant $C_{M}=\lambda^{\frac{1+\alpha d}{p-1}}$ if and only if

$$
\alpha=-\frac{p-1}{2+d(p-1)} .
$$

With this choice, the functionals $\mathcal{F}_{M, \sigma}[u]$ and $\mathcal{I}_{M, \sigma}[u]$ have the same scaling properties, so that we get

$$
\mathcal{J}_{M, \sigma}[u]:=\mathcal{I}_{M, \sigma}[u]-4 \mathcal{F}_{M, \sigma}[u]=\lambda^{\frac{2 p+d(p-1)}{2+d(p-1)}} \sigma^{\frac{d}{2}(1-p)} \mathcal{J}[v]
$$

By expressing the values of $\lambda$ and $\sigma$ in terms of $M$ and $\Theta[u]$, we have that

$$
\begin{gathered}
\lambda=\frac{M}{M_{*}} \text { and } \sigma=\sigma[u]=\left(\frac{M_{*}}{M}\right)^{\frac{2(2-p)-d(1-p)}{2-d(1-p)}} \frac{\Theta[u]}{\Theta_{*}}, \\
\lambda^{\frac{2 p-d(1-p)}{2-d(1-p)}} \sigma^{\frac{d}{2}(1-p)}=\mathrm{h}(M, \Theta[u]) \quad \text { with } \mathrm{h}(M, \Theta):=\left(\frac{M}{M_{*}}\right)^{\frac{d^{2}(1-p)^{2}-2 d\left(p^{2}-4 p+3\right)+4 p}{2(2-d(1-p)]}}\left(\frac{\Theta}{\Theta_{*}}\right)^{\frac{d}{2}(1-p)}
\end{gathered}
$$

We can now rephrase Corollary 12 for a general function $u$ as follows.

Corollary 13. Let $p \in(1,+\infty)$. Assume that $u$ is a nonnegative function in $\mathrm{L}^{1}\left(\mathbb{R}^{d}\right)$ is such that $u^{p}$ and $u|x|^{2}$ are both in $\mathrm{L}^{1}\left(\mathbb{R}^{d}\right)$, and $\nabla u$ is in $\mathrm{L}^{2}\left(\mathbb{R}^{d}\right)$. With the same notations as in Corollary 12 and $\varphi$ defined by (18) we have

$$
\mathcal{J}[u]_{M, \sigma} \geq \mathrm{h}(M, \Theta[u]) \varphi\left(\beta(p-1) \frac{\mathcal{F}_{M, \sigma}[u]}{\mathrm{h}(M, \Theta[u])}\right) \quad \text { with } \quad \sigma=\sigma[u] .
$$


For the same reason as in the fast diffusion case, the choice $\sigma=\sigma[u]$ is remarkable because

$$
\mathcal{F}_{M, \sigma[u]}[u]=\inf _{\sigma>0} \mathcal{F}_{M, \sigma}[u]
$$

so that $\mathfrak{B}_{M, \sigma[u]}$ is the best matching Barenblatt profile, among all Barenblatt profiles with mass $M$ and characteristic scale $\sigma>0$, when measured in relative entropy.

One of the interests of the statement of Corollary 13 is that the free energy $\mathcal{F}_{M, \sigma[u]}[u]$ is an explicit distance to the manifold of the optimal functions for the Gagliardo-Nirenberg inequalities. Variants of the Pinsker-Csiszár-Kullback inequality can be found in Carrillo and Toscani [2000], Del Pino and Dolbeault [2002] and allow to control $\left\|u-\mathfrak{B}_{M, \sigma[u]}\right\|_{\mathrm{L}^{1}\left(\mathbb{R}^{d}\right)}$ under additional assumptions. Here we shall give a simpler result which goes as follows.

Theorem 14. Let $d \geq 1, p \in(1, \infty)$ and assume that $u$ is a non-negative function in $\mathrm{L}^{1}\left(\mathbb{R}^{d}\right)$ such that $u^{p}$ and $x \mapsto|x|^{2} u$ are both integrable on $\mathbb{R}^{d}$. With previous notations, we have that

$$
\mathcal{F}_{M, \sigma[u]}[u] \geq \frac{p}{p-1} \min \{1, p-1\}\left\|u-\mathfrak{B}_{M, \sigma[u]}\right\|_{L^{p}\left(\mathbb{R}^{d}\right)}^{p}
$$

Proof. Let us consider the function

$$
\chi(t):=t^{p}-1-p(t-1)-c_{p}|t-1|^{p}
$$

with $c_{p}:=\min \{1, p-1\}$. The reader is invited to check that for some $t_{p} \in(0,1), \chi^{\prime \prime}(t)<0$ for any $t \in\left(0, t_{p}\right)$, $\chi^{\prime \prime}(t)>0$ for any $t \in\left(t_{p}, 1\right) \cup(1,+\infty)$. Since $\chi(0)=\chi(1)=0$ and $\chi^{\prime}(0)=0$, we get that $\chi(t) \geq 0$ for any $t \geq 0$. As above, let us define $v$ by the rescaling (24) and consider

$$
\mathcal{F}[v]=\frac{1}{p-1} \int_{|x| \leq 1} \chi\left(\frac{v}{\mathfrak{B}}\right) \mathfrak{B}^{p} d x+\frac{1}{p-1} \int_{|x|>1}\left(u^{p}-p\left(1-|x|^{2}\right) u\right) d x
$$

It is straightforward to check that

$$
\mathcal{F}[v]=\frac{c_{p}}{p-1} \int_{|x| \leq 1}|v-\mathfrak{B}|^{p} d x+\frac{1}{p-1} \int_{|x|>1} u^{p} d x \geq c_{p}\|v-\mathfrak{B}\|_{\mathrm{L}^{p}\left(\mathbb{R}^{d}\right)}^{p}
$$

and we conclude using the identities

$$
\mathcal{F}_{M, \sigma[u]}[u]=\mathrm{h}(M, \Theta[u]) \mathcal{F}[v] \quad \text { and } \quad\left\|u-\mathfrak{B}_{M, \sigma[u]}\right\|_{\mathrm{L}^{p}\left(\mathbb{R}^{d}\right)}^{p}=\mathrm{h}(M, \Theta[u])\|v-\mathfrak{B}\|_{\mathrm{L}^{p}\left(\mathbb{R}^{d}\right)}^{p} .
$$

The porous medium equation written in self-similar variables is

$$
\frac{\partial u}{\partial t}=\nabla\left[v \cdot\left(\sigma^{\frac{d}{2}\left(p-p_{c}\right)} \nabla v^{p-1}+2 x\right)\right]=0
$$

and it is equivalent to the porous medium equation $\frac{\partial v}{\partial t}=\Delta v^{p}$ up to a rescaling: as in Dolbeault and Toscani $[2011,2013,2015 \mathrm{~b}]$ we may choose $\sigma=\sigma[u(t, \cdot)]$ to depend on $t$. By using Corollary 13, we get that

$$
\frac{d}{d t} \mathcal{F}_{M, \sigma[u(t, \cdot)]}[u(t, \cdot)]=-\mathcal{I}_{M, \sigma[u(t, \cdot)]}[u(t, \cdot)]
$$

while a direct computation shows that

$$
\frac{d}{d t} \sigma[u(t, \cdot)]=-\frac{2(p-1)^{2}}{p M \Theta\left[\mathfrak{B}_{M, 1}\right]} \sigma[u(t, \cdot)]^{\frac{d}{2}\left(p-p_{c}\right)} \mathcal{F}_{M, \sigma[u(t, \cdot)]}[u(t, \cdot)] \leq 0 .
$$

Altogether this establishes a faster convergence rate of the solutions towards the Barenblatt profiles than one would get using the entropy - entropy production inequality $\mathcal{J}_{M, \sigma[u]}[u] \geq 0$. This is new. More details based on a simpler method will appear in Dolbeault and Toscani [2015b]. 
As a concluding remark, which is valid for the porous medium case, for the fast diffusion case, and also for the linear case of the Ornstein-Uhlenbeck equation, we may observe that two moments are involved while the center of mass $\int_{\mathbb{R}^{d}} x u d x$ is not supposed to be equal to 0 . In that sense our measurement of the distance to the manifold of optimal functions by the deficit functional is an explicit but still a rough estimate, which is definitely not optimal at least in the perturbation regime, that is, close to the optimal functions, which is precisely the regime that was considered in Bianchi and Egnell [1991].

Acknowlegments. This work has been partially supported by the projects STAB, NoNAP and Kibord of the French National Research Agency (ANR). J.D. thanks the Department of Mathematics of the University of Pavia for inviting him and G. Savaré for stimulating discussions. The authors thank F. Bolley for pointing them a missing reference.

(C) 2014 by the authors. This paper may be reproduced, in its entirety, for non-commercial purposes.

\section{References}

A. Arnold and J. Dolbeault. Refined convex Sobolev inequalities. J. Funct. Anal., 225(2):337-351, 2005. ISSN 0022-1236. URL http://dx.doi.org/10.1016/j.jfa.2005.05.003.

A. Arnold, J. A. Carrillo, L. Desvillettes, J. Dolbeault, A. Jüngel, C. Lederman, P. A. Markowich, G. Toscani, and C. Villani. Entropies and equilibria of many-particle systems: an essay on recent research. Monatsh. Math., 142(1-2):35-43, 2004. URL http://dx.doi.org/10.1007/978-3-7091-0609-9_5.

A. Arnold, J.-P. Bartier, and J. Dolbeault. Interpolation between logarithmic Sobolev and Poincaré inequalities. Commun. Math. Sci., 5(4):971-979, 2007. ISSN 1539-6746. URL http://projecteuclid.org/euclid.cms/1199377560.

D. Bakry and M. Ledoux. A logarithmic Sobolev form of the Li-Yau parabolic inequality. Rev. Mat. Iberoam., 22(2):683-702, 2006. ISSN 0213-2230. doi: 10.4171/RMI/470. URL http://dx.doi.org/10.4171/RMI/470.

D. Bakry, I. Gentil, and M. Ledoux. Analysis and geometry of Markov diffusion operators, volume 348 of Grundlehren der Mathematischen Wissenschaften [Fundamental Principles of Mathematical Sciences]. Springer, Cham, 2014. ISBN 978-3-319-00226-2; 978-3-319-00227-9. doi: 10.1007/978-3-319-00227-9. URL http://dx.doi.org/10.1007/978-3-319-00227-9.

J.-P. Bartier and J. Dolbeault. Convex Sobolev inequalities and spectral gap. C. R. Math. Acad. Sci. Paris, 342(5):307-312, 2006. ISSN 1631-073X. doi: 10.1016/j.crma.2005.12.004. URL http://dx.doi.org/10.1016/j.crma.2005.12.004.

J.-P. Bartier, J. Dolbeault, R. Illner, and M. Kowalczyk. A qualitative study of linear drift-diffusion equations with time-dependent or degenerate coefficients. Math. Models Methods Appl. Sci., 17(3):327-362, 2007.

W. Beckner. A generalized Poincaré inequality for Gaussian measures. Proc. Amer. Math. Soc., 105(2):397-400, 1989. ISSN 0002-9939. doi: 10.2307/2046956. URL http://dx.doi.org/10.2307/2046956.

G. Bianchi and H. Egnell. A note on the Sobolev inequality. J. Funct. Anal., 100(1):18-24, 1991. ISSN 0022-1236. doi: 10.1016/0022-1236(91)90099-Q. URL http://dx.doi.org/10.1016/0022-1236 (91) 90099-Q.

A. Blanchet, M. Bonforte, J. Dolbeault, G. Grillo, and J.-L. Vázquez. Asymptotics of the fast diffusion equation via entropy estimates. Archive for Rational Mechanics and Analysis, 191(2):347-385, 02 2009. URL http://dx.doi.org/10.1007/s00205-008-0155-z.

S. Bobkov, N. Gozlan, C. Roberto, and P.-M. Samson. Bounds on the deficit in the logarithmic sobolev inequality. Journal of Functional Analysis, 267(11):4110 - 4138, 2014. ISSN 0022-1236. doi: http://dx.doi.org/10.1016/ j.jfa.2014.09.016. URL http://www.sciencedirect.com/science/article/pii/S0022123614003917.

M. Bonforte, J. Dolbeault, G. Grillo, and J.-L. Vázquez. Sharp rates of decay of solutions to the nonlinear fast diffusion equation via functional inequalities. Proceedings of the National Academy of Sciences, 107(38): 16459-16464, 2010. doi: 10.1073/pnas.1003972107. URL http://dx.doi.org/10.1073/pnas. 1003972107.

M. J. Cáceres, J. A. Carrillo, and J. Dolbeault. Nonlinear stability in $L^{p}$ for a confined system of charged particles. SIAM J. Math. Anal., 34(2):478-494 (electronic), 2002. ISSN 0036-1410. URL http://dx.doi.org/10.1137/S0036141001398435. 
E. A. Carlen. Superadditivity of Fisher's information and logarithmic Sobolev inequalities. J. Funct. Anal., 101(1):194-211, 1991. ISSN 0022-1236. doi: 10.1016/0022-1236(91)90155-X. URL http://dx.doi.org/10.1016/0022-1236(91)90155-X.

E. A. Carlen, R. L. Frank, and E. H. Lieb. Stability estimates for the lowest eigenvalue of a Schrödinger operator. Geom. Funct. Anal., 24(1):63-84, 2014. ISSN 1016-443X. doi: 10.1007/s00039-014-0253-z. URL http://dx.doi.org/10.1007/s00039-014-0253-z.

J. A. Carrillo and G. Toscani. Asymptotic L $\mathrm{L}^{1}$-decay of solutions of the porous medium equation to selfsimilarity. Indiana Univ. Math. J., 49(1):113-142, 2000. ISSN 0022-2518. doi: 10.1512/iumj.2000.49.1756. URL http://dx.doi.org/10.1512/iumj.2000.49.1756.

J. A. Carrillo and G. Toscani. Rényi entropy and improved equilibration rates to self-similarity for nonlinear diffusion equations. Nonlinearity, 27(12):3159-3177, 2014. ISSN 0951-7715. doi: 10.1088/0951-7715/27/12/ 3159. URL http://dx.doi.org/10.1088/0951-7715/27/12/3159.

J. A. Carrillo and J. L. Vázquez. Fine asymptotics for fast diffusion equations. Comm. Partial Differential Equations, 28(5-6):1023-1056, 2003. ISSN 0360-5302. doi: 10.1081/PDE-120021185. URL http://dx.doi.org/10.1081/PDE-120021185.

A. Cianchi, N. Fusco, F. Maggi, and A. Pratelli. The sharp Sobolev inequality in quantitative form. J. Eur. Math. Soc. (JEMS), 11(5):1105-1139, 2009. ISSN 1435-9855. doi: 10.4171/JEMS/176. URL http://dx.doi.org/10.4171/JEMS/176.

I. Csiszár. Information-type measures of difference of probability distributions and indirect observations. Studia Sci. Math. Hungar., 2:299-318, 1967.

M. Del Pino and J. Dolbeault. Best constants for Gagliardo-Nirenberg inequalities and applications to nonlinear diffusions. J. Math. Pures Appl. (9), 81(9):847-875, 2002. ISSN 0021-7824. doi: 10.1016/S0021-7824(02) 01266-7. URL http://dx.doi.org/10.1016/S0021-7824(02)01266-7.

J. Demange. Improved Gagliardo-Nirenberg-Sobolev inequalities on manifolds with positive curvature. J. Funct. Anal., 254(3):593-611, 2008. ISSN 0022-1236. doi: 10.1016/j.jfa.2007.01.017. URL http://dx.doi.org/10.1016/j.jfa.2007.01.017.

J. Denzler, H. Koch, and R. J. McCann. Higher-order time asymptotics of fast diffusion in Euclidean space: a dynamical systems approach. Mem. Amer. Math. Soc., 234(1101):vi+81, 2015. ISSN 0065-9266.

J. Dolbeault and G. Jankowiak. Sobolev and Hardy-Littlewood-Sobolev inequalities. J. Differential Equations, 257(6):1689-1720, 2014. URL http://dx.doi.org/10.1016/j.jde.2014.04.021.

J. Dolbeault and G. Toscani. Fast diffusion equations: matching large time asymptotics by relative entropy methods. Kinetic and Related Models, 4(3):701-716, 2011. URL http://dx.doi.org/10.3934/krm.2011.4.701.

J. Dolbeault and G. Toscani. Improved interpolation inequalities, relative entropy and fast diffusion equations. Ann. Inst. H. Poincaré Anal. Non Linéaire, 30(5):917-934, 2013. ISSN 0294-1449. doi: 10.1016/j.anihpc. 2012.12.004. URL http://dx.doi.org/10.1016/j.anihpc.2012.12.004.

J. Dolbeault and G. Toscani. Best matching Barenblatt profiles are delayed. Journal of Physics A: Mathematical and Theoretical, 48(6):065206, 2015a. URL http://iopscience.iop.org/1751-8121/48/6/065206.

J. Dolbeault and G. Toscani. Nonlinear diffusions: extremal properties of Barenblatt profiles, best matching and delays. Submitted, Jan. 2015b. URL https://hal.archives-ouvertes.fr/hal-01103574.

J. Dolbeault, B. Nazaret, and G. Savaré. On the Bakry-Emery criterion for linear diffusions and weighted porous media equations. Commun. Math. Sci., 6(2):477-494, $2008 . \quad$ URL http://projecteuclid.org/euclid.cms/1214949932.

J. Dolbeault, B. Nazaret, and G. Savaré. A new class of transport distances between measures. Calc. Var. Partial Differential Equations, 34(2):193-231, 2009. ISSN 0944-2669. doi: 10.1007/s00526-008-0182-5. URL http://dx.doi.org/10.1007/s00526-008-0182-5. 
J. Dolbeault, B. Nazaret, and G. Savaré. From Poincaré to logarithmic Sobolev inequalities: a gradient flow approach. SIAM J. Math. Anal., 44(5):3186-3216, 2012. ISSN 0036-1410. doi: 10.1137/110835190. URL http://dx.doi.org/10.1137/110835190.

J. Dolbeault, M. J. Esteban, M. Kowalczyk, and M. Loss. Improved interpolation inequalities on the sphere. Discrete and Continuous Dynamical Systems Series S (DCDS-S), 7(4):695-724, August 2014. URL http://dx.doi.org/10.3934/dcdss.2014.7.695.

M. Fathi, E. Indrei, and M. Ledoux. Quantitative logarithmic Sobolev inequalities and stability estimates. Preprint, Oct. 2014. URL http://arxiv.org/abs/1410.6922.

P. Federbush. Partially alternate derivation of a result of Nelson. J. Mathematical Phys., 10:50-52, 1969. URL http://dx.doi.org/10.1063/1.1664760.

E. Gagliardo. Proprietà di alcune classi di funzioni in più variabili. Ricerche Mat., 7:102-137, 1958. ISSN 0035-5038.

L. Gross. Logarithmic Sobolev inequalities. Amer. J. Math., 97(4):1061-1083, $1975 . \quad$ URL http://www.jstor.org/stable/2373688.

J. Gunson. Inequalities (Birmingham, 1987), volume 129 of Lecture Notes in Pure and Appl. Math., chapter Inequalities in mathematical physics, pages 53-79. Dekker, New York, 1991.

R. Jordan, D. Kinderlehrer, and F. Otto. The variational formulation of the Fokker-Planck equation. SIAM J. Math. Anal., 29(1):1-17, 1998. ISSN 0036-1410. doi: 10.1137/S0036141096303359. URL http://dx.doi.org/10.1137/S0036141096303359.

S. Kullback. On the convergence of discrimination information. IEEE Trans. Information Theory, IT-14: 765-766, 1968. URL http://dx.doi.org/10.1109/TIT.1968.1054195.

R. Latała and K. Oleszkiewicz. Between Sobolev and Poincaré. In Geometric aspects of functional analysis, volume 1745 of Lecture Notes in Math., pages 147-168. Springer, Berlin, 2000. URL http://dx.doi.org/10.1007/BFb0107213.

M. Ledoux. The concentration of measure phenomenon, volume 89 of Mathematical Surveys and Monographs. American Mathematical Society, Providence, RI, 2001. ISBN 0-8218-2864-9. URL http://dx.doi.org/10.1090/surv/089.

M. Ledoux, I. Nourdin, and G. Peccati. Stein's method, logarithmic Sobolev and transport inequalities. ArXiv e-prints, Mar. 2014. URL http://arxiv.org/abs/1403.5855.

E. Mossel and J. Neeman. Robust dimension free isoperimetry in gaussian space. To appear in Annals of Probability, $2014 . \quad$ URL http://www.e-publications.org/ims/submission/index.php/AOP/user/submissionFile/14356? confirm=b4f60ee

L. Nirenberg. On elliptic partial differential equations. Ann. Scuola Norm. Sup. Pisa (3), 13:115-162, 1959. URL https://eudml.org/doc/83226.

F. Otto. The geometry of dissipative evolution equations: the porous medium equation. Comm. Partial Differential Equations, 26(1-2):101-174, 2001. ISSN 0360-5302. URL http://dx.doi.org/10.1081/PDE-100002243.

F. Otto and C. Villani. Generalization of an inequality by Talagrand and links with the logarithmic Sobolev inequality. J. Funct. Anal., 173(2):361-400, 2000. ISSN 0022-1236. doi: 10.1006/jfan.1999.3557. URL http://dx.doi.org/10.1006/jfan.1999.3557.

M. S. Pinsker. Information and information stability of random variables and processes. Translated and edited by Amiel Feinstein. Holden-Day Inc., San Francisco, Calif., 1964.

G. Savaré and G. Toscani. The concavity of Rényi entropy power. IEEE Trans. Inform. Theory, 60(5):2687-2693, 2014. ISSN 0018-9448. doi: 10.1109/TIT.2014.2309341. URL http://dx.doi.org/10.1109/TIT.2014.2309341.

A. J. Stam. Some inequalities satisfied by the quantities of information of Fisher and Shannon. Information and Control, 2:101-112, 1959. ISSN 0890-5401. URL http://dx.doi.org/10.1016/S0019-9958(59)90348-1. 
G. Toscani. An information-theoretic proof of Nash's inequality. Atti Accad. Naz. Lincei Cl. Sci. Fis. Mat. Natur. Rend. Lincei (9) Mat. Appl., 24(1):83-93, 2013. ISSN 1120-6330. doi: 10.4171/RLM/645. URL http://dx.doi.org/10.4171/RLM/645.

G. Toscani. A concavity property for the reciprocal of Fisher information and its consequences on Costa's EPI. ArXiv e-prints, Oct. 2014a. URL http://arxiv.org/abs/1410.2722.

G. Toscani. A strengthened entropy power inequality for log-concave densities. ArXiv e-prints, Aug. $2014 \mathrm{~b}$. URL http://arxiv.org/abs/1408.4049.

C. Villani. A short proof of the "concavity of entropy power". IEEE Trans. Inform. Theory, 46(4):1695-1696, 2000. ISSN 0018-9448. doi: 10.1109/18.850718. URL http://dx.doi.org/10.1109/18.850718.

F. B. Weissler. Logarithmic Sobolev inequalities for the heat-diffusion semigroup. Trans. Amer. Math. Soc., 237:255-269, 1978. ISSN 0002-9947. doi: 10.2307/1997621. URL http://dx.doi.org/10.2307/1997621. 